\title{
Dinâmica da comunidade arbórea de uma floresta semidecidual em Uberlândia, MG, Brasil
}

\author{
Mariana Resende Silva ${ }^{1}$ e Glein Monteiro de Araújo ${ }^{2,3}$
}

Recebido em 13/08/2007. Aceito em 3/04/2008

\begin{abstract}
RESUMO - (Dinâmica da comunidade arbórea de uma floresta semidecidual em Uberlândia, MG, Brasil). O objetivo do trabalho foi de verificar as mudanças ocorridas na comunidade arbórea em um intervalo de 14 anos. O estudo foi realizado em uma área de floresta semidecidual utilizando 50 parcelas de $10 \mathrm{~m} \times 10 \mathrm{~m}$, onde foram amostradas todas as árvores com DAP $\geq 3,18 \mathrm{~cm}$ em 1990 e em 2004 . No primero inventário encontrou-se 95 espécies, 818 indivíduos e área basal de 14,43 $\mathrm{m}^{2}$. No segundo levantamento amostrou-se 95 espécies, 866 indivíduos e área basal de 13,42 $\mathrm{m}^{2}$. No período de 14 anos, 10 espécies desapareceram e o mesmo número ingressou na comunidade. O índice de diversidade de Shannon, na primeira amostragem foi de 4,05 e na segunda 3,72. A taxa média anual de mortalidade foi de $4,1 \%$ e a de recrutamento $4,5 \%$. A meia-vida, o tempo de duplicação, a estabilidade e a reposição, para o número de indivíduos foi 16,92, 15,04, 1,86 e 15,98 anos, respectivamente. O fragmento florestal manteve a riqueza de espécies, porém houve mudanças no índice de diversidade, número de indivíduos e área basal podendo possivelmente ser o resultado do processo de fragmentação e do isolamento dessa comunidade.
\end{abstract}

Palavras-chave: meia-vida, mortalidade, recrutamento, tempo de duplicação, vegetação florestal

ABSTRACT - (Tree community dynamics in a semideciduous forest, in Uberlândia, Minas Gerais State, Brazil). This study describes the dynamics of a tree community based on two successive surveys carried out with a 14 years interval in order to analyze changes in the community structure during the period. The study was done in a semideciduous forest area using 50 plots of $10 \mathrm{~m} \times 10 \mathrm{~m}$, where all trees with DBH $\geq 3.18 \mathrm{~cm}$ were sampled. In 1990 there was registered a total of 95 species, 818 individuals and basal area of $14.43 \mathrm{~m}^{2}$, while in 2004, 95 species, 866 individuals and basal area of the $13.42 \mathrm{~m}^{2}$. The floristic composition changed but the number of species remained same. The Shannon's diversity index was 4.05 and the Pielou's evenness 0.62 in 1990 and 3.72 and 0.57 in 2004 , respectively. The mean annual mortality rate was $4.1 \%$ and the mean annual recruitment rate $4.5 \%$. The half-life, the time duplication, stability and turnover were $16.92,15.04,1.86$ e 15.98 years, respectively. The changes whitch occurred in the forest community shows that this fragment, althought maintaining general structure and species richness, is undergoings changes in diversity and equity which may be still the result of the fragmentation and isolation process.

Key words: forest vegetation, half-life, mortality, recruitment, time duplication

\section{Introdução}

As florestas semideciduais, na região do cerrado, foram intensamente devastadas devido à expansão das atividades agricolas. Atualmente, estas fitofisionomias encontram-se restritas a fragmentos tendo em sua periferia, em geral, lavouras e pastagens (Nascimento et al. 1999).

Nas últimas décadas, houve considerável avanço nos estudos de comunidades florestais, fornecendo informações de suma importância para a realização de ações que visem o adequado manejo, tanto de áreas preservadas, quanto de áreas que sofreram algum tipo de perturbação (Rodrigues et al. 2003). Deste modo, trabalhos foram realizados em florestas semideciduais, investigando a composição florística (Rodrigues et al.
2003), a estrutura fitossociológica (Araújo \& Haridasan 1997) e a estrutura da floresta e as características edáficas (Haridasan \& Araújo 2005). Esses estudos mostram as características estruturais de cada floresta em um dado momento, não abordando investigações em longo prazo sobre a dinâmica dessas fitocenoses. Os estudos de longo prazo verificam as mudanças que ocorrem continuamente na comunidade florestal, revelando aspectos importantes sobre o crescimento, o recrutamento e a mortalidade (Swaine et al. 1990; Felfili 1995).

Trabalhos sobre dinâmica de comunidades florestais foram realizados no Brasil por Werneck et al. (2000), em floresta decidual; Felfili (1995), em floresta de galeria; Lopes \& Schiavini (2007) e em floresta atlântica (Rolim et al. 1999). Trabalhos em florestas semideciduais foram desenvolvidos por Oliveira Filho et al. (1997), Chagas

\footnotetext{
1 Curso de Pós-Graduação em Ecologia e Conservação dos Recursos Naturais

2 Universidade Federal de Uberlândia, Instituto de Biologia, 38400-902 Uberlândia, MG, Brasil

3 Autor para correspondência: glein@ufu.br
} 
et al. (2001), Nascimento et al. (1999) e Paiva et al. (2007). Nestes estudos as elevadas taxas de mortalidade e recrutamento foram atribuídas aos processos de fragmentação e de perturbações sofridas no passado, justificando a importância de pesquisas de longo prazo para detectar as mudanças florísticas e estruturais nessas florestas.

Dessa maneira, a avaliação das mudanças temporais em florestas semideciduais, ajuda a compreender melhor as alterações que ocorrem na comunidade ao longo do tempo e fornece informações sobre o comportamento das espécies, subsidiando ações de restauração e programas de conservação.

O presente estudo teve como objetivo determinar a dinâmica da comunidade arbórea de uma floresta semidecidual em Uberlândia, referente ao período de 1990 a 2004, analisando as mudanças temporais na composição florística, número de indivíduos, área basal, índice de diversidade, taxas de mortalidade e recrutamento, meia vida, tempo de duplicação, estabilidade e reposição.

\section{Material e métodos}

O estudo foi realizado em um fragmento (30 ha) de floresta estacional semidecidual, situado na Fazenda Experimental do Glória (FEG) (1856'57''S e 48 12'14' W), em Uberlândia, Minas Gerais. A floresta semidecidual está adjacente a uma floresta de galeria situada às margens do córrego do Glória. A floresta encontra-se cerca de $2 \mathrm{~km}$ da região urbana de Uberlândia e tem em sua periferia lavouras e pastagens.

O clima da região é do tipo Aw Megatérmico (Köeppen 1948) com verão quente e úmido e inverno frio e seco. A temperatura máxima mensal varia de 27 a $30^{\circ} \mathrm{C}$ e a mínima de 16 a $18{ }^{\circ} \mathrm{C}$. A precipitação anual varia de 1.400 a $1.700 \mathrm{~mm}$ (Rosa et al. 1991). O solo é do tipo Latossolo Vermelho-Escuro originário de sedimentos areno-argilosos provenientes do retrabalhamento do Arenito Bauru. É um solo distrófico, com textura argilosa, tendo saturação de bases de $29 \pm 11 \%$ no horizonte A1 e 7,4 $\pm 3 \%$ no A3 (Haridasan \& Araújo 2005).

Coleta de dados - A primeira amostragem da vegetação arbórea da FEG foi realizada em julho de 1990. Nesse primeiro levantamento (T1) foram amostradas 50 parcelas de $10 \mathrm{~m} \times 10 \mathrm{~m}$, sorteadas em um hectare da floresta. As parcelas foram marcadas com estacas de madeira e delimitadas com fio de náilon resistente a decomposição. Todas as árvores com diâmetro a 1,3 m de altura (DAP) maior ou igual a $3,18 \mathrm{~cm}$ foram marcadas com plaquetas de alumínio numeradas e medidas quanto à altura e ao diâmetro do tronco. As espécies foram identificadas por especialistas ou por comparação com exsicatas existentes no herbário da
Universidade Federal de Uberlândia (HUFU). Os resultados desse primeiro inventário encontram-se em Araújo \& Haridasan (1997).

O segundo levantamento (T2), foi efetuado em julho/2004, (intervalo de 14 anos) quando as parcelas foram reinstaladas. Os procedimentos adotados para a amostragem da vegetação foram os mesmos utilizados no T1. Os indivíduos vivos que não faziam parte do grupo de sobreviventes, ou seja, que não haviam sido registrados em 1990 e apresentaram DAP $\geq 3,18 \mathrm{~cm}$, foram considerados como recrutas ou ingressantes (I) e tiveram suas medidas de diâmetro anotadas e receberam plaquetas de identificação. Novas plaquetas de alumínio foram fixadas em indivíduos amostrados no $\mathrm{T} 1$ quando as primeiras plaquetas foram englobadas pela casca da árvore ou expelidas. Os indivíduos registrados no T1 e não encontrados em T2 foram considerados mortos. A identificação das espécies foi feita no campo e/ou por comparações com exemplares, depositados no Herbário da Universidade Federal de Uberlândia (HUFU).

Com os dados das amostragens em $\mathrm{T} 1$ e $\mathrm{T} 2$ foram calculados os seguintes parâmetros: índices de diversidade de Shannon e eqüidade de Pielou (Magurram 1988), taxa anual de mortalidade (Me), ingresso (Ic), meia-vida [(T(1/2)], tempo de duplicação (TD), reposição $(\mathrm{R})$ e estabilidade $(\mathrm{E})$.

As taxas médias de mortalidade foram calculadas utilizando-se o modelo esponencial: $\mathrm{Me}=-\{\mathrm{Ln}[(\mathrm{N} 1-$ M) $\left.\left.\mathrm{N}^{-1}\right]\right\} \Delta \mathrm{t}^{-1}$, onde, Ln é logaritmo natural na base e, N1 é o número de indivíduos no primeiro levantamento, M é o número de indivíduos mortos e té o intervalo de tempo em anos (14 anos) transcorrido entre os dois levantamentos (Swaine \& Lieberman 1987; Sheil et al. 1995).

Para o cálculo do Ingresso (Ic) ou taxa de recrutamento, a equação utilizada foi: $\mathrm{Ic}=\left\{\left(\mathrm{N} 2 \Delta \mathrm{t}^{-1}\right)-\right.$ Ln [1-(I N2-1)]\} N2-1, onde: N2 o número de indivíduos na segunda amostragem. $\mathrm{O}$ valor de I corresponde ao número de indivíduos ingressantes. Quando o cálculo utiliza valores em área basal, o I é substituído pelo valor da área basal ganha (ABg) (Sheil \& May 1996).

A meia vida (T[1/2]), que corresponde ao tempo necessário para a comunidade reduzir seu tamanho à metade, mantida a atual taxa de mortalidade, foi obtida utilizando-se a fórmula: $\mathrm{T}(1 / 2)=\mathrm{Ln}(0,5) \mathrm{Me}^{-1}$ (Korning \& Balslev 1994). O tempo de duplicação (TD) que é o tempo necessário para que a comunidade dobre seu tamanho, mantida a presente taxa de ingresso, foi calculado utilizando-se o seguinte modelo matemático: $\mathrm{TD}=\mathrm{Ln}(2) \operatorname{Ln}(1+\mathrm{Ic})^{-1}$ (Korning \& Balslev 1994).

Foram calculadas a estabilidade (E) e a reposição (R) em número de indivíduos e em área basal, utilizando as equações: $\mathrm{E}=\left|\mathrm{T}(1 / 2)-\mathrm{T}_{2}\right|$ e $\mathrm{R}=\left(\mathrm{T}(1 / 2)+\mathrm{T}_{2}\right) 2^{-1}$, respectivamente (Korning \& Balslev 1994). Aárea é mais 
estável quando o valor obtido for mais próximo de zero; e quanto menor o valor de reposição mais dinâmica é a comunidade (Korning \& Balslev 1994).

Confecção dos histogramas - Para se obter os intervalos de classes para a montagem dos histogramas de diâmetro utilizou-se a seguinte fórmula: $\mathrm{A} / \mathrm{K}$, onde A representa a amplitude dos valores de diâmetro e $\mathrm{K}$ indica uma constante definida pelo algoritmo de Sturges, que consiste em: $1+3,3 \times \log _{10} \mathrm{n}$, onde, $\mathrm{n}$ é o número total de indivíduos amostrados (Bonini \& Bonini 1972). O resultado dessa razão $(\mathrm{A} / \mathrm{K})$ representa o valor do incremento utilizado para definir os intervalos de classes. Para todas as classes diamétricas foram calculados os valores absolutos e as taxas médias anuais de mortalidade e ingresso, área basal acrescentada e área basal perdida.

Análise estatística - Para comparar os índices de diversidade de Shannon entre T1 e T2 foi utilizado o teste "t" de Hutcheson. Para verificar se houve diferença significativa na freqüência de indivíduos nas diferentes classes de diâmetro entre $\mathrm{T} 1$ e T2, entre o T2 e o número de mortos e entre o T2 e o número de ingressantes, foi aplicado o teste de Kolmogorov-Smirnov (Sieguel 1975).

\section{Resultados e discussão}

Composição florística e índice de diversidade - O número total de espécies registradas nos dois levantamentos na floresta semidecidual da FEG foi 105, sendo obtidas em T1, 95 espécies, pertencentes a 45 famílias e 83 gêneros (Araújo \& Haridasan 1997) e em T2, 95 espécies, distribuídas em 43 famílias e 81 gêneros. A composição florística da floresta semidecidual mudou em 14 anos. Dez das espécies encontradas em T1 não foram registradas em T2. Destas espécies, oito apresentaram um ou dois indivíduos. No segundo levantamento foram amostradas dez espécies novas, destas, nove recrutaram um indivíduo cada e uma dois indivíduos (Tab. 1).

Geralmente, a perda e ganho de espécies em dinâmica das florestas tropicais estão relacionadas com

Tabela 1. Estrutura e dinâmica da comunidade arbórea, expressa em número de indivíduos e área basal, na floresta semidecidual da Fazenda Esperimental do Glória, Uberlândia, MG, Brasil, em ordem descrescente do número de indivíduos em N1. Números de indivíduos: N1 = inicial (1990); Nd = mortalidade; $\mathrm{Nr}=$ recrutamento e N2 = final (2004). Área basal: Ab1 = inicial (1990); Abm = mortos; Abi = ingressos; Abg $=$ acrescentada; Ab2 = final (2004).

\begin{tabular}{|c|c|c|c|c|c|c|c|c|c|}
\hline & \multicolumn{4}{|c|}{ Número de indivíduos } & \multicolumn{5}{|c|}{ Área basal } \\
\hline & N1 & $\mathrm{Nd}$ & $\mathrm{Nr}$ & $\mathrm{N} 2$ & $\mathrm{Ab} 1$ & Abm & Abi & Abg & $\mathrm{Ab} 2$ \\
\hline Casearia grandiflora Camb. & 40 & 29 & 34 & 45 & 0,224 & 0,162 & 0,070 & 0,107 & 0,169 \\
\hline Siparuna guianensis Aubl. & 38 & 29 & 64 & 73 & 0,074 & 0,060 & 0,121 & 0,139 & 0,152 \\
\hline Aspidosperma discolor A. DC. & 34 & 5 & 17 & 46 & 0,356 & 0,039 & 0,029 & $-0,028$ & 0,29 \\
\hline Cryptocaria aschersoniana $\mathrm{Mez}$ & 33 & 8 & 7 & 32 & 0,087 & 0,087 & 0,001 & 0,001 & 0,001 \\
\hline Virola sebifera Aubl. & 30 & 12 & 10 & 28 & 0,281 & 0,107 & 0,020 & 0,224 & 0,398 \\
\hline Cheiloclinium cognatum (Miers) A.C. Smith & 27 & 6 & 9 & 30 & 0,025 & 0,010 & 0,000 & 0,005 & 0,02 \\
\hline Astronium nelson-rosae D.A. Santin & 26 & 6 & 5 & 25 & 0,205 & 0,017 & 0,008 & 0,135 & 0,323 \\
\hline Pouteria torta (Mart.) Radlk. & 26 & 3 & 5 & 28 & 0,469 & 0,097 & 0,036 & 0,274 & 0,646 \\
\hline Ixora warmingii Müll. Arg. & 25 & 12 & 9 & 22 & 0,119 & 0,038 & 0,033 & 0,094 & 0,175 \\
\hline Casearia grandiflora Camb. & 40 & 29 & 34 & 45 & 0,224 & 0,162 & 0,070 & 0,107 & 0,169 \\
\hline Apuleia leiocarpa (Vog.) Macbr. & 21 & 11 & 3 & 13 & 0,464 & 0,206 & 0,005 & 0,032 & 0,290 \\
\hline Cordia sellowiana Cham. & 21 & 10 & 2 & 13 & 0,011 & 0,007 & 0,002 & 0,004 & 0,008 \\
\hline Machaerium villosum Vog. & 21 & 6 & 2 & 17 & 0,000 & 0,000 & 0,001 & 0,001 & 0,001 \\
\hline Ocotea corymbosa (Meisn.) Mez & 21 & 11 & 0 & 10 & 2,028 & 0,707 & 0,000 & 0,069 & 1,390 \\
\hline Siphoneugenia densiflora Berg & 20 & 2 & 8 & 26 & 0,100 & 0,020 & 0,018 & 0,035 & 0,114 \\
\hline Trichilia pallida $\mathrm{Sw}$. & 20 & 3 & 89 & 106 & 0,067 & 0,024 & 0,140 & 0,183 & 0,225 \\
\hline Copaifera langsdorffii Desf. & 18 & 10 & 0 & 8 & 0,261 & 0,082 & 0,004 & 0,061 & 0,240 \\
\hline Duguetia lanceolata A. St.-Hil. & 18 & 5 & 33 & 46 & 0,047 & 0,005 & 0,060 & 0,150 & 0,191 \\
\hline Terminalia glabrescens Mart. & 18 & 8 & 0 & 10 & 0,451 & 0,175 & 0,000 & $-0,041$ & 0,235 \\
\hline Protium heptaphyllum (Aubl.) March. & 17 & 5 & 12 & 24 & 0,342 & 0,072 & 0,030 & 0,140 & 0,410 \\
\hline Casearia sylvestris $\mathrm{Sw}$. & 16 & 12 & 3 & 7 & 0,090 & 0,065 & 0,005 & $-0,007$ & 0,018 \\
\hline Alibertia sessilis (Vell.) K. Schum. & 14 & 7 & 1 & 8 & 0,047 & 0,017 & 0,001 & 0,000 & 0,030 \\
\hline Matayba guianensis Aubl. & 14 & 9 & 2 & 7 & 0,074 & 0,041 & 0,003 & 0,024 & 0,056 \\
\hline Agonandra brasiliensis Miers ex Benth. \& Hook. F. & 13 & 3 & 0 & 10 & 0,112 & 0,012 & 0,000 & 0,016 & 0,116 \\
\hline Luehea paniculata Mart. & 13 & 6 & 0 & 7 & 0,114 & 0,058 & 0,000 & 0,020 & 0,076 \\
\hline Pouteria gardneri (Mart. \& Miq.) Baehni & 13 & 6 & 2 & 9 & 0,234 & 0,073 & 0,007 & 0,063 & 0,224 \\
\hline Aspidosperma subincanum Mart. & 12 & 11 & 0 & 1 & 0,142 & 0,103 & 0,000 & $-0,023$ & 0,017 \\
\hline Callisthene major Mart. & 11 & 6 & 2 & 7 & 0,230 & 0,046 & 0,011 & 0,102 & 0,286 \\
\hline Garcinia gardneriana (Planch. \& Triana) Zappi & 10 & 5 & 2 & 7 & 0,028 & 0,012 & 0,02 & 0,022 & 0,038 \\
\hline
\end{tabular}


Tabela 1 (continuação)

\begin{tabular}{|c|c|c|c|c|c|c|c|c|c|}
\hline & \multicolumn{4}{|c|}{ Número de indivíduos } & \multicolumn{5}{|c|}{ Área basal } \\
\hline & N1 & $\mathrm{Nd}$ & $\mathrm{Nr}$ & $\mathrm{N} 2$ & $\mathrm{Ab} 1$ & Abm & Abi & $\mathrm{Abg}$ & $\mathrm{Ab} 2$ \\
\hline Inga sessilis (Vell.) Mart. & 9 & 5 & 4 & 8 & 0,064 & 0,049 & 0,008 & 0,022 & 0,037 \\
\hline Platycyamus regnellii Benth. & 9 & 3 & 0 & 6 & 0,391 & 0,008 & 0,000 & 0,079 & 0,463 \\
\hline Cariniana estrellensis (Raddi) O. Kuntze & 8 & 1 & 7 & 14 & 0,678 & 0,002 & 0,013 & 0,444 & 1,119 \\
\hline Coussarea contracta (Walp.) Müll. Arg. & 8 & 5 & 2 & 5 & 0,037 & 0,037 & 0,005 & 0,005 & 0,005 \\
\hline Hirtella gracilipes (Hook. f.) Prance & 8 & 0 & 1 & 9 & 0,060 & 0,000 & 0,001 & 0,032 & 0,093 \\
\hline Cupania vernalis Camb. & 7 & 7 & 1 & 1 & 0,141 & 0,022 & 0,011 & 0,028 & 0,146 \\
\hline Miconia sellowiana Naud. & 7 & 6 & 1 & 2 & 0,130 & 0,092 & 0,001 & 0,014 & 0,052 \\
\hline Qualea jundiahy Warm. & 7 & 3 & 1 & 5 & 0,318 & 0,019 & 0,004 & 0,082 & 0,381 \\
\hline Vitex polygama Cham. & 7 & 1 & 1 & 7 & 0,360 & 0,111 & 0,016 & 0,061 & 0,310 \\
\hline Amaioua intermedia Mart. & 6 & 4 & 2 & 4 & 0,028 & 0,014 & 0,002 & 0,011 & 0,024 \\
\hline Cardiopetalum calophyllum Schltdl. & 6 & 5 & 5 & 6 & 0,011 & 0,009 & 0,007 & 0,007 & 0,009 \\
\hline Guettarda viburnoides Cham. \& Schltdl. & 6 & 4 & 1 & 3 & 0,012 & 0,010 & 0,002 & 0,006 & 0,008 \\
\hline Jacaranda macrantha Cham. & 6 & 4 & 2 & 4 & 0,150 & 0,067 & 0,037 & 0,024 & 0,107 \\
\hline Margaritaria nobilis L. f. & 6 & 4 & 0 & 2 & 0,088 & 0,036 & 0,000 & 0,004 & 0,056 \\
\hline Miconia cecidophora Naud. & 6 & 1 & 0 & 5 & 0,019 & 0,002 & 0,000 & 0,020 & 0,036 \\
\hline Piptocarpha macropoda (DC.) Backer & 6 & 5 & 1 & 2 & 0,258 & 0,225 & 0,004 & 0,023 & 0,056 \\
\hline Tapirira obtusa ((Benth.) D.J. Mitch. & 6 & 2 & 3 & 7 & 0,303 & 0,108 & 0,038 & 0,223 & 0,418 \\
\hline Aegiphila sellowiana Cham. & 5 & 1 & 0 & 4 & 0,180 & 0,040 & 0,000 & 0,052 & 0,192 \\
\hline Campomanesia velutina Berg. & 5 & 4 & 0 & 1 & 0,067 & 0,047 & 0,000 & 0,000 & 0,021 \\
\hline Heisteria ovata Benth. & 5 & 1 & 5 & 9 & 0,038 & 0,012 & 0,015 & 0,051 & 0,077 \\
\hline Simira viridiflora Steyerm. & 5 & 0 & 4 & 9 & 0,127 & 0,000 & 0,009 & 0,028 & 0,155 \\
\hline Coccoloba mollis Casar. & 4 & 1 & 0 & 3 & 0,858 & 0,253 & 0,000 & 0,186 & 0,792 \\
\hline Faramea cyanea Müell. Arg. & 4 & 1 & 0 & 3 & 0,042 & 0,001 & 0,000 & 0,011 & 0,053 \\
\hline Machaerium nictitans Benth. & 4 & 4 & 0 & 0 & 0,033 & 0,017 & 0,000 & $-0,013$ & 0,003 \\
\hline Ocotea spixiana (Nees) Mez & 4 & 2 & 1 & 3 & 0,103 & 0,031 & 0,029 & 0,065 & 0,137 \\
\hline Pera glabrata (Schott) Poepp. ex Baill. & 4 & 3 & 0 & 1 & 0,323 & 0,318 & 0,000 & 0,000 & 0,005 \\
\hline Sloanea monosperma Vell. & 4 & 3 & 0 & 1 & 0,013 & 0,008 & 0,000 & 0,001 & 0,006 \\
\hline Cassia ferruginea Scharad. ex DC. & 3 & 2 & 0 & 1 & 0,332 & 0,125 & 0,000 & 0,078 & 0,284 \\
\hline Schefflera morototoni (Aubl.) Mag., Steyerm. & 3 & 0 & 3 & 6 & 0,156 & 0,000 & 0,004 & 0,297 & 0,452 \\
\hline Hymenaea courbaril $\mathrm{L}$. & 3 & 0 & 1 & 4 & 0,104 & 0,000 & 0,001 & 0,015 & 0,120 \\
\hline Maclura tinctoria D. Don. ex Steud. & 3 & 2 & 0 & 1 & 0,819 & 0,162 & 0,095 & 0,120 & 0,777 \\
\hline Myrciaria glanduliflora (Kiaerst) Mattos \& Legr. & 3 & 1 & 4 & 6 & 0,004 & 0,003 & 0,004 & 0,005 & 0,007 \\
\hline Sorocea bonplandii (Baill.) W. Burg. & 3 & 0 & 2 & 5 & 0,012 & 0,000 & 0,003 & 0,001 & 0,013 \\
\hline Handroanthus serratifolius (Vahl) S.O. Grose & 3 & 2 & 0 & 1 & 0,015 & 0,013 & 0,000 & $-0,001$ & 0,001 \\
\hline Zanthoxylum rhoifolium Lam. & 3 & 3 & 0 & 0 & 0,067 & 0,067 & 0,000 & 0,000 & 0,000 \\
\hline Albizia polycephala (Benth.) Killip & 2 & 0 & 2 & 4 & 0,012 & 0,000 & 0,003 & 0,006 & 0,017 \\
\hline Aspidosperma cylindrocarpon Müll. Arg. & 2 & 2 & 1 & 1 & 0,005 & 0,005 & 0,001 & 0,001 & 0,001 \\
\hline Bauhinia ungulata L. & 2 & 1 & 0 & 1 & 0,007 & 0,002 & 0,000 & 0,003 & 0,008 \\
\hline Celtis iguanaea (Jacq.) Sarg. & 2 & 1 & 2 & 3 & 0,006 & 0,001 & 0,006 & 0,004 & 0,008 \\
\hline Inga laurina $(\mathrm{Sw}$.$) Willd.$ & 2 & 0 & 0 & 2 & 0,008 & 0,000 & 0,000 & $-0,001$ & 0,008 \\
\hline Maprounea guianensis Aubl. & 2 & 2 & 0 & 0 & 0,080 & 0,080 & 0,000 & 0,000 & 0,000 \\
\hline Maytenus sp. & 2 & 2 & 0 & 0 & 0,021 & 0,007 & 0,000 & $-0,014$ & 0,000 \\
\hline Myrcia tomentosa (Aubl.) DC. & 2 & 1 & 0 & 1 & 0,006 & 0,001 & 0,000 & 0,003 & 0,007 \\
\hline Nectandra membranacea (Sw.) Griseb. & 2 & 2 & 4 & 4 & 0,120 & 0,120 & 0,010 & 0,010 & 0,010 \\
\hline Sweetia fruticosa Spreng. & 2 & 0 & 0 & 2 & 0,006 & 0,000 & 0,000 & 0,003 & 0,009 \\
\hline Actinostemon concepcionis Müll. Arg. & 1 & 1 & 4 & 4 & 0,001 & 0,001 & 0,007 & 0,007 & 0,007 \\
\hline Annona cacans Warm. & 1 & 1 & 3 & 3 & 0,001 & 0,001 & 0,012 & 0,012 & 0,012 \\
\hline Aspidosperma parvifolium A. DC. & 1 & 1 & 0 & 0 & 0,002 & 0,002 & 0,000 & 0,000 & 0,000 \\
\hline Byrsonima laxiflora Griseb. & 1 & 1 & 0 & 0 & 0,003 & 0,003 & 0,000 & 0,000 & 0,000 \\
\hline Casearia decandra Jacq. & 1 & 1 & 0 & 0 & 0,010 & 0,010 & 0,000 & 0,000 & 0,000 \\
\hline Cedrela fissilis Vell. & 1 & 1 & 1 & 1 & 0,007 & 0,009 & 0,003 & 0,005 & 0,003 \\
\hline Croton floribundus Spreng. & 1 & 1 & 1 & 1 & 0,409 & 0,034 & 0,024 & 0,203 & 0,578 \\
\hline Diospyros hispida A. DC. & 1 & 0 & 0 & 1 & 0,012 & 0,000 & 0,000 & 0,000 & 0,013 \\
\hline Eriotheca candolleana (K. Schum.) A. Robyns & 1 & 0 & 0 & 1 & 0,030 & 0,000 & 0,000 & 0,001 & 0,031 \\
\hline Guarea guidonia L. Sleumer & 1 & 1 & 0 & 0 & 0,005 & 0,005 & 0,000 & 0,000 & 0,000 \\
\hline Guazuma ulmifolia Lam. & 1 & 0 & 0 & 1 & 0,023 & 0,000 & 0,000 & $-0,002$ & 0,021 \\
\hline Myrsine umbellata Mart. & 1 & 0 & 0 & 1 & 0,002 & 0,000 & 0,000 & 0,000 & 0,001 \\
\hline Ormosia fastigiata Tul. & 1 & 0 & 0 & 1 & 0,002 & 0,000 & 0,000 & 0,001 & 0,002 \\
\hline Ouratea castaneifolia (DC.) Engl. & 1 & 0 & 0 & 1 & 0,004 & 0,000 & 0,000 & 0,000 & 0,004 \\
\hline
\end{tabular}




\begin{tabular}{|c|c|c|c|c|c|c|c|c|c|}
\hline & \multicolumn{4}{|c|}{ Número de indivíduos } & \multicolumn{5}{|c|}{ Área basal } \\
\hline & $\mathrm{N} 1$ & $\mathrm{Nd}$ & $\mathrm{Nr}$ & $\mathrm{N} 2$ & $\mathrm{Ab} 1$ & $\mathrm{Abm}$ & Abi & $\mathrm{Abg}$ & $\mathrm{Ab} 2$ \\
\hline Prockia crucis P. Browne ex L. & 1 & 0 & 1 & 2 & 0,009 & 0,000 & 0,001 & 0,007 & 0,016 \\
\hline Psidium rufum Mart. ex DC. & 1 & 0 & 1 & 2 & 0,001 & 0,000 & 0,002 & 0,003 & 0,005 \\
\hline Qualea dichotoma (Mart.) Warm. & 1 & 1 & 0 & 0 & 0,158 & 0,115 & 0,000 & $-0,043$ & 0,000 \\
\hline Roupala brasiliensis Klotzsch & 1 & 0 & 1 & 2 & 0,001 & 0,000 & 0,005 & 0,005 & 0,006 \\
\hline Rudgea viburnoides (Cham.) Benth. & 1 & 0 & 0 & 1 & 0,002 & 0,000 & 0,000 & 0,004 & 0,006 \\
\hline Tapirira guianensis Aubl. & 1 & 0 & 0 & 1 & 0,046 & 0,000 & 0,000 & 0,018 & 0,064 \\
\hline Vochysia tucanorum Mart. & 1 & 0 & 0 & 1 & 0,006 & 0,000 & 0,000 & 0,004 & 0,010 \\
\hline Xylopia sericea St.-Hil. & 1 & 1 & 0 & 0 & 0,013 & 0,013 & 0,000 & 0,000 & 0,000 \\
\hline Casearia gossypiosperma Briq. & 0 & 0 & 2 & 2 & 0,000 & 0,000 & 0,003 & 0,003 & 0,003 \\
\hline Eugenia sp. & 0 & 0 & 1 & 1 & 0,000 & 0,000 & 0,001 & 0,001 & 0,001 \\
\hline Ficus sp. & 0 & 0 & 1 & 1 & 0,000 & 0,000 & 0,002 & 0,002 & 0,002 \\
\hline Machaerium stipitatum DC. Vog. & 0 & 0 & 1 & 1 & 0,391 & 0,391 & 0,000 & 0,000 & 0,00 \\
\hline Myrcia splendens (Sw.) DC. & 0 & 0 & 1 & 1 & 0,000 & 0,000 & 0,001 & 0,001 & 0,001 \\
\hline Myrcia sp. & 0 & 0 & 1 & 1 & 0,000 & 0,000 & 0,001 & 0,001 & 0,001 \\
\hline Ocotea lanceolata Nees & 0 & 0 & 1 & 1 & 0,000 & 0,000 & 0,001 & 0,001 & 0,001 \\
\hline Ocotea percoriacea (Meisn.) Kosterm. & 0 & 0 & 1 & 1 & 0,000 & 0,000 & 0,005 & 0,005 & 0,005 \\
\hline Pavonia malacophylla (Link \& Otto) Garcke & 0 & 0 & 1 & 1 & 0,000 & 0,000 & 0,001 & 0,001 & 0,001 \\
\hline Unonopsis lindmanii R.E. Fries & 0 & 0 & 1 & 1 & 0,000 & 0,000 & 0,001 & 0,001 & 0,001 \\
\hline
\end{tabular}

as menos abundantes na comunidade (Nascimento et al. 1999; Werneck et al. 2000), como ocorrido no presente estudo. As espécies que saíram e as ingressantes na amostragem tinham poucos indivíduos (um a dois), característica comum em 25 a 33\% das espécies de florestas tropicais (Lang \& Knight 1983; Swaine et al. 1987) e próximo ao valor encontrado em florestas semideciduais, 33\% por Araújo \& Haridasan (1997), em Uberlândia e 40\% por Paula et al. (2004), em Viçosa, ambos em Minas Gerais. As espécies com poucos indivíduos podem sofrer extinção local devido a flutuações ambientais e reaparecer em outros levantamentos por migração, recrutamento de indivíduos do banco de sementes, plântulas ou pelo crescimento de jovens que não atingiram o critério mínimo de inclusão (Swaine et al. 1987). O tamanho reduzido, o isolamento e a interferência antrópica na floresta podem determinar o desaparecimento de algumas espécies ou a redução de suas populações (Paiva et al. 2007), favorecendo o crescimento de outras espécies.

O índice de diversidade de Shannon em T1 $(4,05)$ foi maior que em $\mathrm{T} 2(3,72)(\mathrm{t}=6,45 ; \mathrm{gl}=1576,92$; $\mathrm{p}<0,001)$. O índice de eqüidade de Pielou foi de 0,62 em T1 e de 0,57 em T2. Esta diferença ocorreu porque Siparuna guianensis e Trichilia pallida apresentaram população numerosa em T2. Em outras formações florestais brasileiras as mudanças na riqueza e na densidade das espécies foram pequenas e não alteraram os índices de diversidade durante o período analisado (Appolinário et al. 2005; Paiva et al. 2007). Estudos em florestas tropicais indicam que em áreas não atingidas por distúrbios severos e livres de interferência antrópica ocorrem poucas mudanças na diversidade, sugerindo uma aparente estabilidade (Swaine et al. 1987).

Número de indivíduos e área basal - O número de árvores amostradas no primeiro levantamento (T1) e no segundo (T2) foram de 818 e 866 indivíduos, respectivamente. Entre os dois censos, houve um ganho líquido de 48 indivíduos, resultado da morte de 357 e do recrutamento de 405 . A área basal total da comunidade nos dois inventários foi $14,43 \mathrm{~m}^{2} \mathrm{em} \mathrm{T} 1 \mathrm{e} 13,42 \mathrm{~m}^{2}$, em T2 (Tab. 2). As árvores mortas neste período representaram uma perda de $5,05 \mathrm{~m}^{2}$. O recrutamento e o crescimento dos sobreviventes contribuíram com $1,03 \mathrm{~m}^{2}$ e $3,01 \mathrm{~m}^{2}$, respectivamente, para o aumento em área basal. Os fragmentos de florestas semideciduais

Tabela 2. Dinâmica da comunidade arbórea com valores referentes ao número de indivíduos e área basal na floresta semidecidual da Fazenda Experimental do Glória, Uberlândia, MG, Brasil.

\begin{tabular}{lcc}
\hline Características & $\begin{array}{c}\text { Número de } \\
\text { indivíduos }\end{array}$ & $\begin{array}{c}\text { Área basal } \\
\left(\mathrm{m}^{2}\right)\end{array}$ \\
\hline Amostragem no T1 & 818 & 14,43 \\
Amostragem no T2 & 866 & 13,42 \\
Mortos & 357 & 5,05 \\
Recrutas & 405 & 1,03 \\
Incremento em área basal & - & 4,04 \\
Taxa Anual de Mortalidade (\%) & 4,10 & 3,08 \\
Taxa Anual de Ingresso (\%) & 4,50 & 2,56 \\
Meia Vida (anos) & 16,92 & 22,53 \\
Tempo de Duplicação (anos) & 15,04 & 27,44 \\
Reposição (anos) & 15,98 & 24,98 \\
Estabilidade (anos) & 1,86 & 4,91 \\
\end{tabular}


tropicais, em geral encontram-se em desequilíbrio quanto às taxas de mortalidade e recrutamento, ganho e perda de biomassa (Appolinário et al. 2005; Oliveira Filho et al. 2007). No presente estudo o desequilíbrio no número de indivíduos entre $\mathrm{T} 1$ e T2 deve-se principalmente ao crescimento do número de indivíduos da população de Trichilia pallida e Siparuna guianensis, espécies tolerantes à sombra (Oliveira Filho et al. 1997; Appolinário et al. 2005) e que parecem ser boas competidoras nesse ambiente. Por outro lado, a morte de espécies de dossel como Ocotea corymbosa e Terminalia glabrescens contribuiu para a perda de biomassa da comunidade arbórea. A morte de indivíduos dessas espécies pode ter ocorrido devido a sua sensibilidade à presença de densa cobertura de cipós em suas copas e de cupinzeiros na base do tronco, observados no primeiro levantamento.

$\mathrm{O}$ maior número de indivíduos ocorreu na primeira classe de diâmetro nos dois levantamentos, sendo maior em T2 (Fig. 1A). Não houve diferença (KS para duas amostras, $\left.\chi^{2}=6,79, \mathrm{gl}=2, \mathrm{p}>0,05\right)$ quanto ao número de indivíduos nas diversas classes de diâmetro entre o T1 e o T2, na comunidade arbórea da FEG. A morte de árvores, a quebra de galhos e, como consequência, a formação de clareiras abrem novos espaços para recrutas resultando em maior número de indivíduos com menor diâmetro. Tal fato proporciona distribuição de freqüências na forma exponencial, comum em florestas tropicais naturais onde as árvores com menor diâmetro geralmente possuem maior população (Hartshorn 1980; Swaine et al. 1987). A distribuição da área basal por classe de diâmetro, em geral foi maior no T1. Em T2 o alto valor de área basal para a classe de $59-66 \mathrm{~cm}$ foi devido ao crescimento de espécies sobreviventes de dossel (Fig. 1B).

Mortalidade - Dos 818 indivíduos amostrados em T1, 357 morreram até 2004, cerca de 51 árvores mortas $\mathrm{ha}^{-1}$ ano $^{-1}$, resultando em uma taxa média anual de mortalidade de $4,1 \%$ (Tab. 2). Essa taxa é considerada elevada quando comparada com grandes áreas preservadas de floresta tropical, com valores que variam de 1 a $2 \%$ ao ano (Lieberman \& Lieberman 1987; Swaine et al. 1987). Em florestas brasileiras preservadas, foi determinado um percentual médio anual de $1,5 \%$, em floresta semidecidual em Linhares (Rolim et al. 1999) e em floresta galeria em Brasília 3,5\% (Felfili 1995). Em florestas semideciduais alteradas, em Lavras e Piracicaba, foram determinadas taxas $2,6 \%$ e $4,5 \%$, respectivamente (Oliveira Filho et al. 1997; Nascimento et al. 1999).

As altas taxas de mortalidade podem ser resultados de mudanças globais, principalmente o aumento de $\mathrm{CO}_{2}$ (Phillips 1996), alterações microclimáticas (Laurance et al.1998) e resultado do efeito de fragmentação de hábitats (Nascimento et al. 1999). No presente estudo, os efeitos da fragmentação podem ter sido um dos fatores responsáveis pela elevada taxa de mortalidade, uma vez que a floresta semidecidual da FEG é isolada por lavouras e pastagem e próxima ao perímetro urbano de Uberlândia.

Ocorreu diferença significativa, quanto ao número de indivíduos mortos entre os diversos intervalos de classes de diâmetro (KS para uma amostra, $\mathrm{D}_{\text {máx }}=0,553$, $\mathrm{n}=357, \mathrm{p}<0,01)$. O maior número de indivíduos mortos foi registrado na classe de 3,0-10 cm (Fig. 1C). Segundo Felfili (1995), as maiores taxas de mortalidade ocorrem em indivíduos das menores classes diamêtricas devido à baixa capacidade de competição em relação às árvores situadas no dossel. Entretanto, em florestas onde foram detectados distúrbios mais severos durante o monitoramento, nota-se maior taxa de mortalidade para árvores grandes (Rolim et al. 1999).

Na FEG as espécies que mais contribuíram para a mortalidade nas duas primeiras classes de diâmetro foram Siparuna guianensis, secundária inicial encontrada no sub-bosque, Casearia grandiflora e C. sylvestris, ambas pioneiras, sendo uma de dossel intermediário e a outra de sub-bosque, respectivamente. Siparuna guianensis e C. grandiflora, apesar de perderem 29 e 40 indivíduos, recrutaram 64 e 34, respectivamente, ao contrário, de C. sylvestris que apresentou 12 indivíduos mortos e apenas três ingressaram na população (Tab. 1). A alta mortalidade e o baixo recrutamento de $C$. sylvestris indicam baixa capacidade competitiva dos indivíduos jovens no local de estudo.

A área basal perdida foi maior na classe de $31-38 \mathrm{~cm}$ (Fig. 1D). Neste intervalo, as espécies que mais contribuíram para a perda em área basal foram Machaerium nictitans que perdeu todos os seus indivíduos no período de 14 anos e Ocotea corymbosa que teve sua área basal reduzida devido à mortalidade de 11 indivíduos (Tab. 1). Essas espécies dependem de muita luz (Oliveira Filho et al. 1997) e a cobertura de suas copas com cipós, como foi observado no T1, possivelmente contribuiu para a morte dos indivíduos.

Recrutamento - Dos 866 indivíduos amostrados em T2, 405 eram recrutas (Tab. 2), cerca de 58 árvores recrutadas ha ${ }^{-1}$ ano $^{-1}$, resultando em um taxa média anual de recrutamento de $4,5 \%$. Uma das maiores taxas de recrutamento registrada em floresta semidecidual alterada, foi de 4,04\%, em um intervalo de 10 anos (Paiva et al. 2005). Por outro lado taxas de 1,8 a $2,99 \%$ foram encontradas em intervalos de 5 a 7 anos nesse mesmo tipo fisionômico de floresta (Oliveira Filho et al. 1997; Appolinário et al. 2005; Oliveira Filho et al. 2007).

Cinquenta e quatro espécies $(56,3 \%$ do total de espécies) presentes em 1990 apresentaram recrutamento até 2004 (Tab. 1). As taxas anuais de recrutamento para as espécies variaram de 0 a 14,95\%. Para 40 espécies 
as taxas de recrutamento ocorreram entre $1-10 \%$ e apenas quatro espécies tiveram taxas maiores que $10 \%$. As maiores taxas de recrutamento foram obtidas para S. guianensis (14,95\%), Trichilia pallida $(13,07 \%)$ e Cardiopetalum calophyllum $(12,80 \%)$. A maior taxa média anual de recrutamento $(4,5 \%)$ ocorreu principalmente devido ao ingresso de T. pallida (89 indivíduos) e $S$. guianensis (64), espécies comuns no sub-bosque de florestas semidecíduas e Casearia grandiflora (34), espécie de dossel intermediário.

Houve diferença quanto ao número de indivíduos recrutados entre os diversos intervalos de classe (KS para uma amostra, $\left.\mathrm{D}_{\text {máx }}=0,765, \mathrm{n}=405, \mathrm{p}<0,01\right)$. O maior recrutamento ocorreu na classe de diâmetro de
3,0-10 cm (Fig. 1E), representando 98,54\% do total de indivíduos ingressos e, consequientemente, a maior taxa de ingresso também ocorreu nesta classe $\left(7,23 \%\right.$ ano $\left.^{-1}\right)$. Essas espécies com menor diâmetro ocupam o subbosque da floresta e parecem ser favorecidas por Copaifera langsdorffii, Machaerim villosum, Cariniana estrellensis e Ocotea corymbosa que ocupam o dossel superior da floresta (Araújo \& Haridasan 1977).

A área basal acrescentada foi maior no intervalo 59-66 cm devido ao crescimento em diâmetro dos indivíduos sobreviventes (Fig. 1F). Neste intervalo, as espécies que mais contribuíram para o acréscimo em área basal foram Cassia ferruginea, Ocotea corymbosa, Cariniana estrellensis e Qualea jundiahy, enquanto a

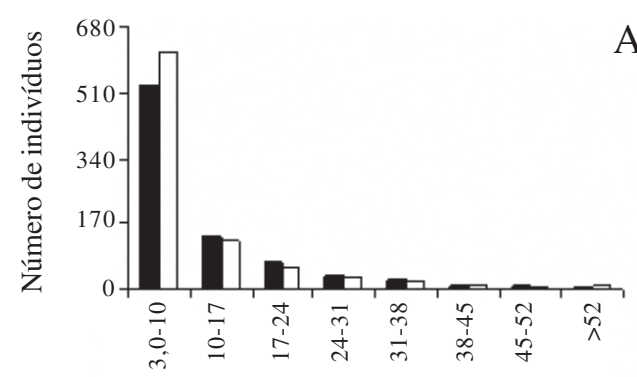

C

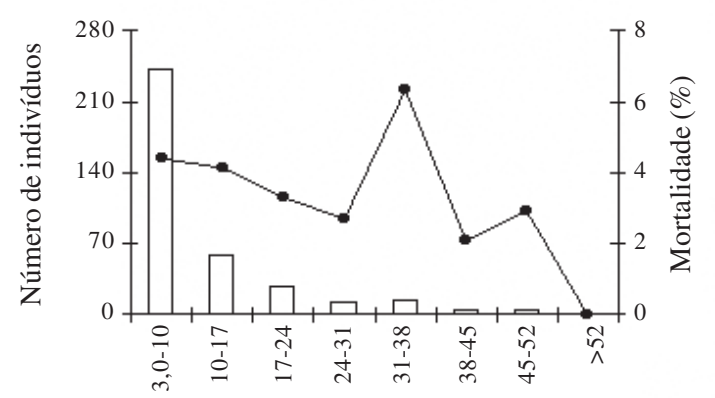

$\mathrm{E}$

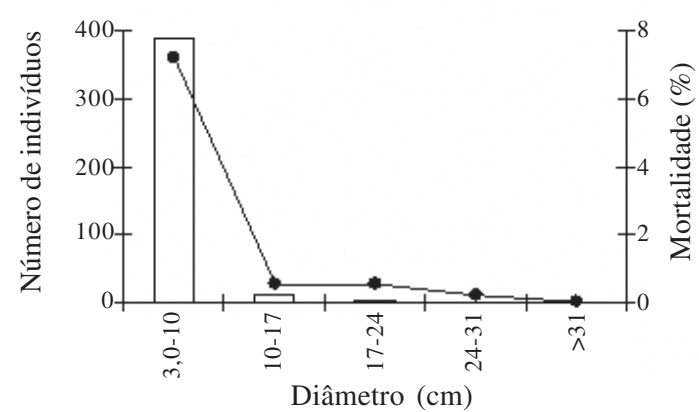

A

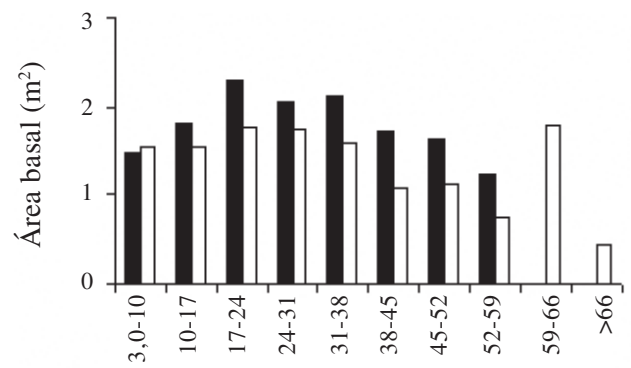

D

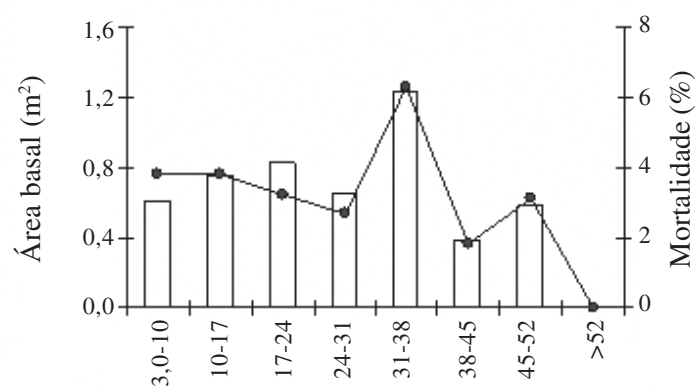

F

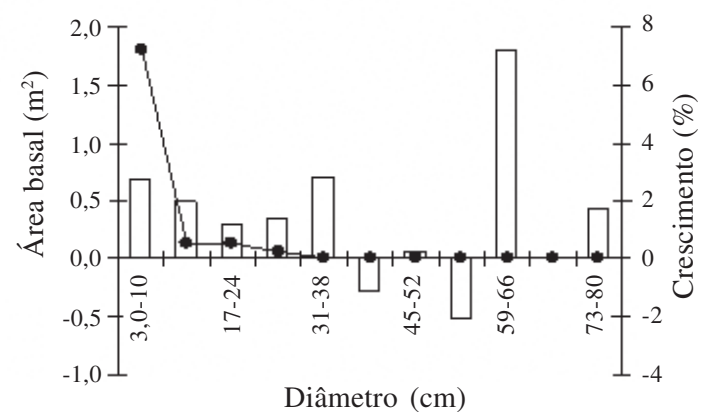

Figura 1. Mudanças na composição florística e na estrutura da comunidade de espécies arbóreas em diferentes classes de diâmetro, na floresta semidecidual da Fazenda Esperimental do Glória, Uberlândia, MG, Brasil. A. Distribuição dos indivíduos ( $\bullet=$ T1 (1990); $\square=$ T2 (2004). B. Área basal ( $=$ T1 (1989); $\square=$ T2 (2000). C. Mortalidade de indivíduos ( $\square=$ indivíduos mortos; - $\bullet-=$ porcentagem de mortalidade). D. Perda de área basal ( $\square=$ área basal perdida; - $\bullet-=$ porcentagem de mortalidade). E. Número de indivíduos recrutados $(\square=$ número de ingressos; $-\bullet-=$ taxa anual de ingresso). F. Acréscimo em área basal ( $\square=$ crescimento; - $\bullet-=$ porcentagem de crescimento). 
maior taxa de ingresso ocorreu na classe de 3,0-10 cm.

Meia vida, tempo de duplicação, estabilidade e reposição O valor de meia vida (16,9 anos), quanto ao número de indivíduos (Tab. 2), encontrado na floresta da FEG foi inferior aos registrados, no mesmo tipo fisionômico de vegetação, por Oliveira Filho et al. (1997), Guilherme et al. (2004) e Paiva et al. (2007). Por outro lado, o tempo de duplicação registrado aqui (15,04 anos, Tab. 2) situou-se entre os valores (12,3 a 23,6 anos) encontrados pelos mesmos autores. O menor valor de meia vida indicaria que o fragmento florestal da FEG é mais dinâmico, pelo menos no período de estudo, que as outras florestas semideciduais estudadas. Quando uma floresta apresenta taxas de mortalidade, de recrutamento, meiavida e tempo de duplicação equilibradas, como no presente estudo, encontra-se em estado silvigênico de equilíbrio (Korning \& Balslev 1994).

A comunidade da florestal da FEG é mais estável em relação ao número de indivíduos (1,06 anos) do que em relação à área basal $(4,91$ anos, Tab. 2). Para reposição é mais dinâmica, em relação ao número de indivíduos $(15,98)$ do que em relação à área basal $(24,98)$. Com relação a outros fragmentos de florestas semideciduais, possui estabilidade e dinamismo semelhante àquela estudada por Paiva et al. 2007 e maior do que as amostradas por Oliveira Filho et al. (1997) e Guilherme et al. (2004).

\section{Agradecimentos}

A Adenilza Borges, pela ajuda na amostragem da vegetação em 2004; a Adriana Arantes, pela identificação de espécies da família Myrtaceae.

\section{Referências bibliográficas}

Appolinário, V.; Oliveira Filho, A.T. \& Guilherme, F.A.G. 2005. Tree population and community dynamics in a Brazilian tropical semideciduous forest. Revista Brasileira de Botânica 28: 347-360.

Araújo, G.M. \& Haridasan, M. 1997. Estrutura fitossociológica de duas matas mesófilas semideciduas, em Uberlândia, Triângulo Mineiro. Naturalia 22: $115-129$.

Bonini, E.E. \& Bonini, S.E. 1972. Estatística teórica e exercícios. São Paulo, Editora Loyola.

Chagas, R.K.; Oliveira Filho, A.T.; van den Berg, E. \& Scolforo, J.R.S. 2001. Dinâmica de populações arbóreas em um fragmento de floresta estacional semidecidual montana em Lavras, Minas Gerais. Revista Árvore 25: 39-57.

Felfili, M.J. 1995. Growth, recruitment and mortality in the Gama gallery Forest in central Brazil over a six-year period (1985-1991). Journal of Tropical Ecology 11: 67-83.

Guilherme, F.A.G.; Oliveira Filho, A.T.; Appolinário, V. \& Bearzoti, E. 2004. Effects of flooding regime and woody bamboos on tree community dynamics in a section of tropical semideciduous forest in South-Eastern Brazil. Plant Ecology 174: 19-36.
Haridasan, M. \& Araújo, G.M. 2005. Perfil nutricional de espécies lenhosas de duas florestas semideciduas em Uberlândia, MG. Revista Brasileira de Botânica 28: 295-303.

Hartshorn, G.S. 1980. Neotropical forest dynamics. Biotropica 12: 23-30.

Köeppen, W. 1948. Climatologia: con un estudio de los climas de la tierra. Mexico, Fondo de Cultura Economica.

Korning, J. \& Balslev, H. 1994. Growth and mortality of trees in Amazonian tropical rain forest in Ecuador. Journal of Vegetation Science 4: 77-86.

Lang, G.E. \& Knight, D.H. 1983. Tree growth, mortality, recruitment, and canopy gap formation during a 10-year period in a tropical moist forest. Ecology 64: 1075-1080.

Laurance, W.F.; Ferreira, J.M.; Rankin-de-Merona, J.M. \& Laurance, S.G. 1998. Rain Forest fragmentation and the dynamics of Amazonian tree communities. Ecology 19: 2032-2040.

Lieberman, D. \& Lieberman, M. 1987. Forest tree growth and dynamics at La Selva, Costa Rica (1969-1982). Journal of Tropical Ecology 3: 347-358.

Lopes, S.F. \& Schiavini, I. 2007. Dinâmica da comunidade arbórea de mata de galeria da Estação Ecológica do Panga, Minas Gerais, Brasil. Acta Botanica Brasilica 21: 249-261.

Magurram, A.E. 1988. Ecological diversity and its measurements. New Jersey, Princeton University.

Nascimento, H.E.M.; Dias, A.S.; Tabanez, A.A.J. \& Viana, V.M. 1999. Estrutura e dinâmica de populações arbóreas de um fragmento de floresta estacional semidecidual na região de Piracicaba, SP. Revista Brasileira de Biologia 59: $329-342$

Oliveira Filho, A.T.; Mello, J.M. \& Scolforo, J.R.S. 1997. Effects of past disturbance and edges on tree community structure and dynamics within a fragment of tropical semideciduous forest in south-eastern Brazil over a fiveyear period (1987-1992). Plant Ecology 131: 45-66.

Oliveira Filho, A.T.; Carvalho, W.A.C.; Machado, E.L.M.; Higuchi, P.; Castro, G.C.; Silva, A.C.; Santos, R.M.; Borges, L.F.R.; Corrêa, B.S. \& Alves, J.M. 2007. Dinâmica da comunidade e populações arbóreas da borda e interior de um remanescente florestal na serra da Mantiqueira, Minas Gerais, em um intervalo de cinco anos (1999-2004). Revista Brasileira de Botânica 30: $149-161$

Paiva, L.V.; Araújo, G.M. \& Pedroni, F. 2007. Struture and dynamics of a woody plant community of a tropical semi-deciduous seasonal forest in the "Estação Ecológica do Panga", municipality of Uberlândia, Minas Gerais, Brasil. Revista Brasileira de Botânica 30: 365-373.

Paula, A.; Silva, A.F.; Marco Júnior, P.; Santos, F.A.M. \& Souza, A.L. 2004. Sucessão ecológica da vegetação arbórea em uma Floresta Estacional semidecidual, Viçosa, MG, Brasil. Acta Botanica Brasilica 18: 407-423.

Phillips, O.L. 1996. Long-term environmental changes in tropical forests: increasing tree turnover. Enviromental Conservation 23: 235-248.

Rodrigues, L.A.; Carvalho, D.A.; Oliveira Filho, A.T.; Botrel, R.T. \& Silva, E.A. 2003. Florística e estrutura da comunidade arbórea de um fragmento florestal em Luminárias, MG. Acta Botanica Brasilica 17: 71-87.

Rolim, S.G.; Couto, H.T.Z. \& Jesus, R.M. 1999. Mortalidade e recrutamento de árvores na Floresta Atlântica em Linhares (ES). Scientia Forestalis 55: 49-69.

Rosa, R.; Lima, S.C. \& Assunção, W.L. 1991. Abordagem preliminar das condições climáticas de Uberlândia. Sociedade \& Natureza 3: 91-108.

Sheil, D.; Burslem, D.F.R.P. \& Alder, D. 1995. The interpretation and misinterpretation of mortality rate measures. Journal of Ecology 83: 331-333.

Sheil, M.D. \& May, R.M. 1996. Mortality and recruitment rate evaluations in heterogeneous tropical forests. Journal of Ecology 84: 91-100.

Sieguel, S. 1975. Estatística não paramétrica: para as ciências do comportamento. São Paulo, McGraw-Hil do Brasil Ltda.

Swaine, M.D.; Lieberman, D. \& Hall, J.B. 1990. Structure and dynamics a tropical dry forest in Ghana. Vegetation 88: 31-51.

Swaine, M.D.; Lieberman, D. \& Putz, F.E. 1987. The dynamics of tree populations in tropical forest: a review. Journal of Tropical Ecology 3 : 359:366.

Werneck, M.S.; Franceschinelli, E.V. \& Tameirão Neto, E. 2000. Mudanças florísticas e estrutura de uma floresta decídua durante um período de quatro anos (1994-1998), na região do Triângulo Mineiro, MG. Revista Brasileira de Botânica 23: 399-411.

Versão eletrônica do artigo em www.scielo.br/abb e http://www.botanica.org.br/acta/ojs 